\title{
Understanding the Solar Sources of In Situ Observations
}

\author{
Pete Riley, Zoran Mikic, and Jon Linker \\ Science Applications International Corporation, San Diego, California.
}

Thomas H. Zurbuchen

University of Michigan, Ann Arbor, Michigan.

\begin{abstract}
The solar wind can, to a good approximation be described as a two-component flow with fast, tenuous, quiescent flow emanating from coronal holes, and slow, dense and variable flow associated with the boundary between open and closed magnetic fields. In spite of its simplicity, this picture naturally produces a range of complex heliospheric phenomena, including the presence, location, and orientation of corotating interaction regions and their associated shocks. In this study, we apply a two-step mapping technique, incorporating a magnetohydrodynamic model of the solar corona, to bring in situ observations from Ulysses, WIND, and ACE back to the solar surface in an effort to determine some intrinsic properties of the quasi-steady solar wind. In particular, we find that a "layer" of $\sim 35,000 \mathrm{~km}$ exists between the Coronal Hole Boundary (CHB) and the fast solar wind, where the wind is slow and variable. We also derive a velocity gradient within large polar coronal holes (that were present during Ulysses' rapid latitude scan) as a function of distance from the CHB. We find that $v=713 \mathrm{~km} / \mathrm{s}+3.2 d$, where $d$ is the angular distance from the CHB boundary in degrees.
\end{abstract}

\section{INTRODUCTION}

Early in situ observations in the vicinity of Earth established that the solar wind consisted primarily of two components: slow $(\sim 300 \mathrm{~km} / \mathrm{s})$, dense, and cool wind; and fast $(\sim 700 \mathrm{~km} / \mathrm{s})$, tenuous, and hot wind (1). The relatively quiescent properties of the high-speed wind observed by Ulysses $(2,3,4)$ suggest that this is the basic equilibrium state of the solar wind. The origin of the slow solar wind and its variability, on the other hand, remain poorly understood.

More recently, the idea that there might exist more than two basic types of solar wind has been raised within a number of contexts (e.g., 5, 6). While there may be merit to such views, in this study we limit ourselves to the bimodal viewpoint for simplicity. To support this, in Figure 1 we show Ulysses plasma and composition measurements from launch through the present. Density (logarithmic) runs along the x-axis, speed runs along the y-axis, and temperature (logarithmic) runs along the $\mathrm{z}$-axis.

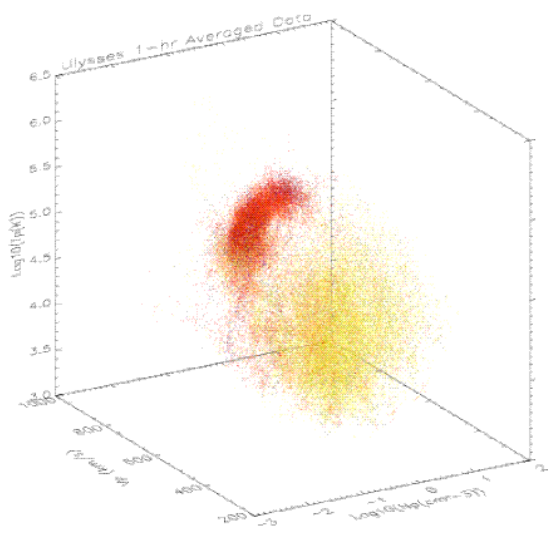

FIGURE 1. Ulysses 1-hr averaged measurements of density $(\mathrm{x})$, speed $(\mathrm{y})$, and proton temperature $(\mathrm{z})$, color-coded according to the ratio 07/O6 (red indicating the lowest values and yellow indicating the highest values). 
The points have been color-coded according to the ratio of $\mathrm{O} 7$ to $\mathrm{O} 6$ (7). While there is room in such a plot to hide additional intrinsic components, clearly the dominate flow can be described as bimodal.

In this report, we map in situ data back to the Sun to uncover some of the intrinsic properties and gradients associated with the solar wind. We begin with a brief summary of the mapping procedure, then summarize our main results, and conclude with a discussion of their implications.

\section{MAPPING PROCEDURE}

A number of techniques have been developed to connect in situ observations with various solar features. Typically this involves some kind of mapping procedure to extrapolate the solar wind observations from the point of measurement back to the Sun. These range from the simplest "constant speed," or "ballistic" mapping (e.g., 8) where it assumed that the plasma maintains constant radial velocity from the Sun to the point of observation) to more sophisticated global, time-dependent MHD simulations that can trace field lines all the way from the solar surface to the point of observation (9). We have investigated the errors associated with several of these approaches and find that a combination of the ballistic mapping, together with a realistic MHD model for the coronal magnetic field typically provides the best compromise (10). The ballistic mapping is used to bring the data from the point of observation (1-5 AU) to $30 R_{\mathrm{s}}$ where the dominant variation is with respect to longitude. Between 1 and $30 R_{\mathrm{s}}$, on the other hand, latitudinal variations are most significant and the MHD model is used to trace along magnetic field lines back to the solar surface.

In figure 2 we use a global MHD model to illustrate the errors introduced by the ballistic mapping procedure. The two curves compare the MHD solution at $30 R_{\mathrm{s}}$ with the MHD solution at $1 \mathrm{AU}$, which has been ballistically mapped back to $30 R_{\mathrm{s}}$. Differences of $5^{\circ}-10^{\circ}$ are apparent, and represent the limit in longitudinal accuracy for the mapping process. While one might suppose that 3-D MHD solutions driven by solar observations would provide a more accurate way to map the in situ data, relatively small errors of $\sim 50$ $\mathrm{km} / \mathrm{s}$ in the solution at $1 \mathrm{AU}$ lead to longitudinal offsets of $\sim 8^{\circ}$. Although the heliospheric MHD models have been very successful as a tool to investigate the large-scale structure of the solar wind (e.g., 9,11) improved specification of the boundary conditions will be necessary before they can be used as a reliable mapping tool.

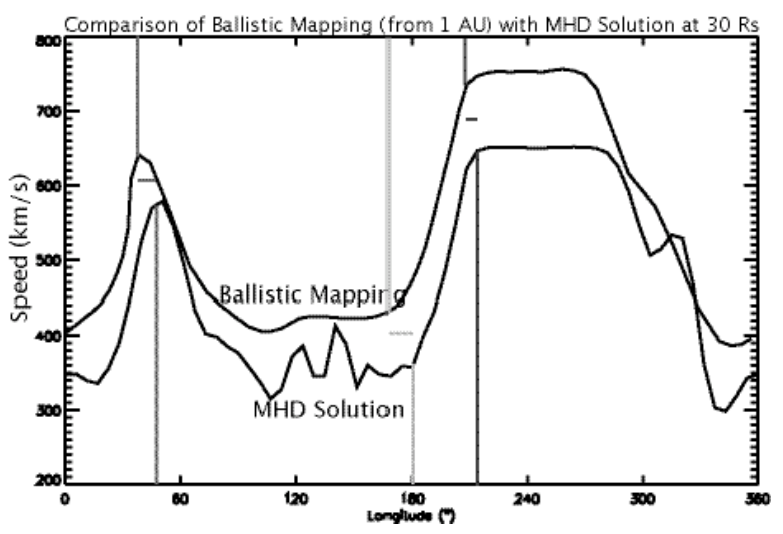

FIGURE 2. Comparison of the ballistic mapping with the MHD solution at $30 R_{\mathrm{s}}$. Vertical lines and short horizontal bars indicate differences in longitude for 3 distinct points in the stream profile.

\section{RESULTS}

We illustrate the mapping technique in Figure 3, which combines data and modeling results. The closed regions represent coronal holes, as computed by the coronal MHD solution for this time period (Carrington Rotation (CR) 1953 - beginning August 18 ${ }^{\text {th }}, 1999$ ). These are often similar, but not identical to the coronal holes computed from Kitt Peak He I 1083 nm observations (typically being a superset) and are colorcoded according to the underlying (highly smoothed) observed photospheric fields. Superimposed onto this are the trajectories of $\mathrm{ACE}$ and Ulysses. At the time ACE was located at $1 \mathrm{AU}$ and 6.6 degrees above the heliographic equator. Ulysses was located at $4.66 \mathrm{AU}$ and $\sim 32.2^{\circ} \mathrm{S}$. The starting point of the trajectories (already mapped to $30 R_{\mathrm{S}}$ ) are straight-line traces moving from right to left (with increasing time) and all points map back to origins within coronal holes (by definition). These mappings have been color-coded according to the measured polarity of the interplanetary magnetic field. Given the approximations in the mapping procedure and the relatively high level of solar activity during this time period, the strong correlation between the mapped in situ polarities and the polarity of the coronal holes is quite remarkable, providing strong support that the mapping procedure has been successful.

Once the plasma data have been mapped back to the solar surface, we can investigate its relationship with various solar features. For this study, we focus on the speed of the solar wind and its distance to the computed Coronal Hole Boundary (CHB). In Figure 4 we have calculated the minimum distance to the $\mathrm{CHB}$ for each plasma measurement. These observations were made at the minimum of the solar cycle, when 
large, long-lived polar coronal holes were present (CR 1913, beginning August 22 $\left.2^{\text {nd }}, 1996\right)$. During this time Ulysses was completing its first sampling of the northern polar coronal hole and was returning toward the ecliptic.

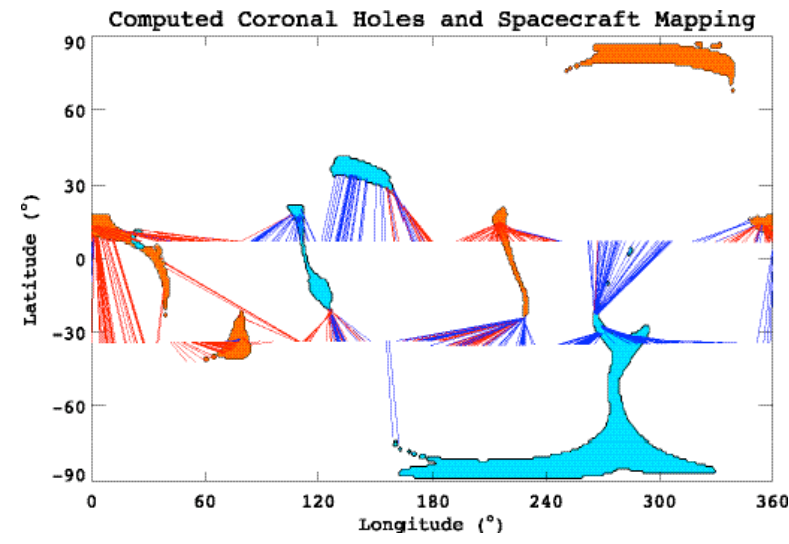

FIGURE 3. ACE (closest to equator) and Ulysses trajectories mapped back to the Sun for CR 1953. Coronal holes are colored according to the observed photospheric field. Trajectories are color-coded according to the measured in situ polarity of the IMF (red/blue corresponds to outward/inward polarity).

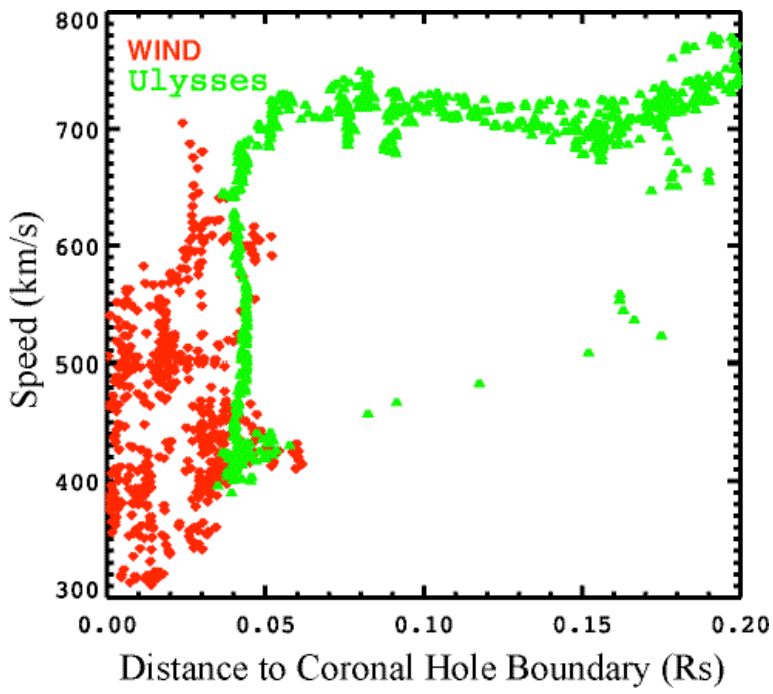

FIGURE 4. Mapped speed as a function of distance to the CHB for CR 1913. Ulysses measurements are shown in green and WIND measurements are shown in red.

We infer a "boundary layer" of $\sim 0.05 R$, corresponding to $\sim 35,000 \mathrm{~km}$ over which the solar wind is slow and variable, and beyond which the wind is fast and relatively steady. This scale size matches well with the average size of a supergranule $(32,000$ $\mathrm{km})$. While this profile tends to be common during solar minimum conditions, near solar maximum it is rarely, if ever present. As an example we return to CR 1953 and in Figure 5 we again display speed as a function of the distance to the CHB.

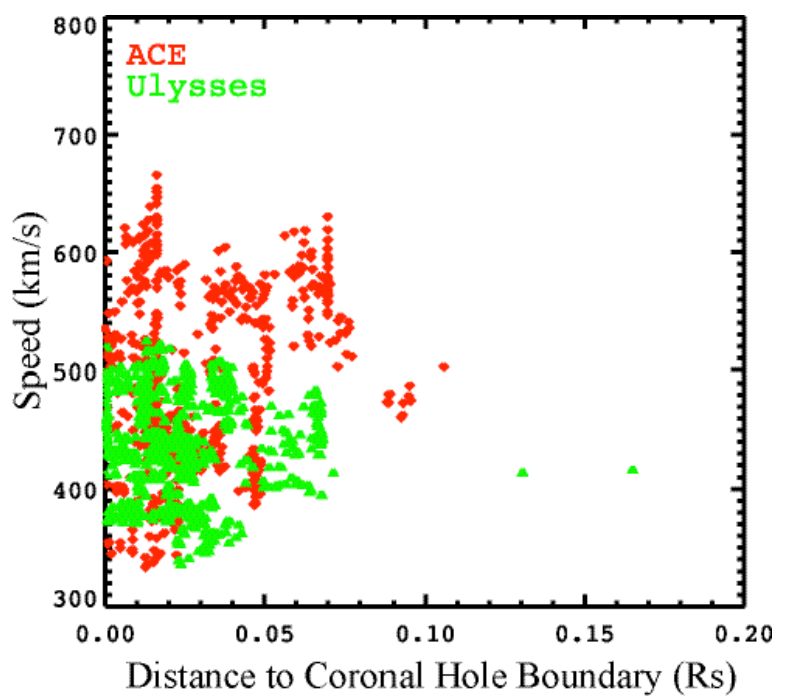

FIGURE 5. Mapped speed as a function of distance to the CHB for CR 1953. Ulysses measurements are shown in green and $\mathrm{ACE}$ measurements are shown in red.

Although there is a tendency for the wind speed to increase with distance away from the $\mathrm{CHB}$, there is no obvious sign of a "layer" of slow wind, at least as far as $0.07 R_{\mathrm{S}}$. However, as can be seen from Figure 3 during this time period, there were no extensive coronal holes that were intercepted by the trajectory of either spacecraft.

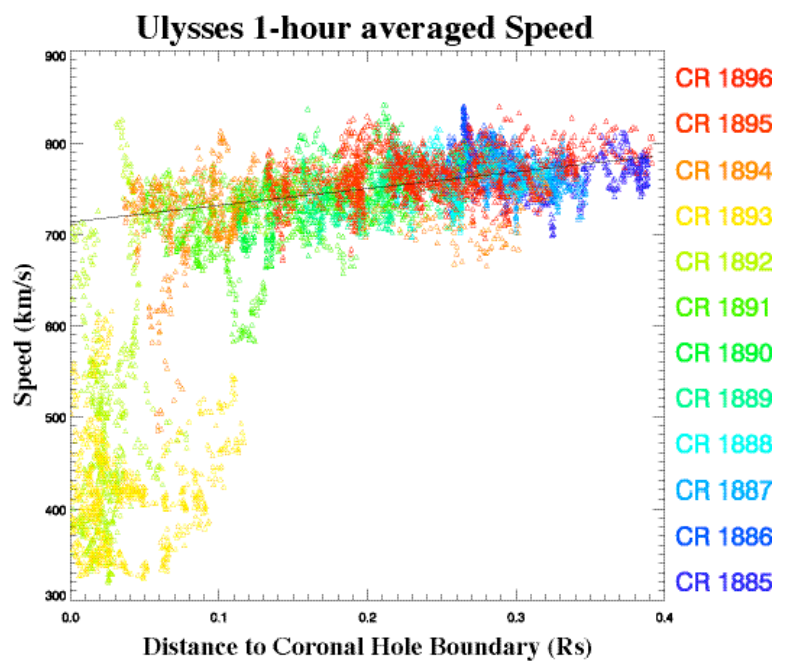

FIGURE 6. Solar wind speed versus distance to the coronal hole boundary for 12 Carrington rotations occurring during Ulysses rapid latitude scan. The solid line represents a leastsquares fit to speeds above $600 \mathrm{~km} / \mathrm{s}$. 
To investigate possible gradients deeper within coronal holes, we mapped data from 12 solar rotations during Ulysses' so-called rapid latitude scan (which occurred during the declining phase of the solar cycle) and computed the distance to the CHB. These results are shown in Figure 6. The distance to the CHB can be interpreted either in units of $R_{\mathrm{S}}$ or Sun-centered radians $\left(1 R_{\mathrm{S}}=1\right.$ radian $)$. Thus the $\mathrm{x}$-axis covers a range of $23^{\circ}$, indicating that Ulysses became deeply immersed within the coronal holes. Again, note that most of the slow wind tends to fall within $\sim 0.05 R_{\mathrm{S}}$ of the CHB. Beyond this, the wind tends to be fast and relatively steady. A least squares fit to data above 600 $\mathrm{km} / \mathrm{s}$ emphasizes the relatively constant positive gradient in speed with respect to distance to the CHB.

\section{DISCUSSION}

The least squares fit to the data in Figure 6 gives the following relationship between distance to the CHB $(d)$ and speed:

$$
v=713 k m s^{\square 1}+3.2 d\left(^{\circ}\right)
$$

Or, recasting it in units of solar radii (or equivalently radians):

$$
v=713 k m s^{\square 1}+183.7 d\left(R_{s}\right)
$$

These results may be contrasted with previous work (4) which found a mean high-speed flow of 703 $\mathrm{km} / \mathrm{s}$ and a latitudinal gradient of $0.950 \mathrm{~km} /$ degree. Our results suggest a gradient that is $\sim 3.4$ times larger. We suggest that equation (1) is closer to the true speed gradient, at least within large polar coronal holes and that the lower gradient in the previous study (4) reflects the approximate correlation of distance into a coronal hole with increasing heliographic latitude. Since even large polar CHBs can vary significantly in latitude, as a function of longitude, however, the intrinsic gradient is masked, and the apparent disagreement is thus a "dilution" effect. By the same token, the CHBs computed in the MHD models, are only approximately correct and equations (1) and (2) should be viewed as lower limits to the true gradient.

\section{ACKNOWLEDGMENTS}

The authors gratefully acknowledge the support of the national Aeronautics and Space Administration (SEC-GI, SEC-TP, SR\&T, and LWS programs). We also thank the National Science Foundation at the San
Diego Supercomputer Center for providing computational support.

\section{REFERENCES}

1. Neugebauer, M., and C. W. Snyder, Mariner 2 observations if the solar wind. I. Average properties, $J$. Geophys. Res. 71, 4469, 1966.

2. Riley, P., S. J. Bame, B. L. Barraclough, W. C. Feldman, J. T. Gosling, G. W. Hoogeveen, D. J. McComas, J. 1. Phillips, B. E. Goldstein, and M. Neugebauer, Ulysses solar wind plasma observations at high latitudes, $A d v$. Space Res., 20, 15, 1997.

3. McComas, D. J., S. J. Bame, B. L. Barraclough, W. C. Feldman, H. O. Funsten, J. T. Gosling, P. Riley, R. Skoug, A. Balogh, R. J. Forsyth, B. E. Goldstein, and M. Neugebauer, Ulysses rerun to the slow solar wind, Geophys, Res. Lett., 25, 1, 1998.

4. McComas D. J., B. L. Barraclough, H. O. Funsten, J. T. Gosling, E. Santiago Munoz, R. M. Skoug, B. E. Goldstein, M. Neugebauer, P. Riley, A. Balogh, Solar wind observations over Ulysses' first full polar orbit, $J$. Geophys. Res., 105, 10419, 2000.

5. Neugebauer, M., P. C. Liewer, E. J. Smith, R .M. Skoug, T. H. Zurbuchen, Sources of the Solar Wind at Solar Activity Maximum, submitted to J. Geophys. Res., 2002.

6. McComas D. J., H. A. Elliott, and R. von Steiger, Solar wind from high-latitude coronal holes at solar maximum, Geophys. Res., Lett., in press, 2002.

7. von Steiger R., J. Geiss, and G. Gloeckler, Composition of the Solar Wind, in Cosmic Winds and the Heliosphere edited by J. R. Jokipii, C. P. Sonett and M. S. Giampapa, Tucson, Arizona, The University of Arizona Press, pp. 581-616, 1997.

8. Nolte J. T. and E. C. Roelof, Large-Scale Structure of the Interplanetary Medium, Solar Physics 33, 483, 1973.

9. Riley P., J. A. Linker, and Z. Mikic, An empiricallydriven global MHD model of the solar corona and inner heliosphere J. Geophys. Res., 106, 15889, 2001.

10. Neugebauer M., R. J. Forsyth, A. B. Galvin, K. L. Harvey, J. T. Hoeksema, A J. Lazarus, R. P. Lepping, J. A. Linker, Z. Mikic, J. T. Steinberg, R. von Steiger, Y.M. Wang, and R. F. Wimmer-Schweingruber, Spatial structure of the solar wind and comparisons with solar data and models, J. Geophys. Res., 103, 14587, 1998.

11. Riley, P., J. A. Linker, and Z. Mikic, Modeling the heliospheric current sheet: Solar-cycle variations, $J$. Geophys. Res., 107, 10.1029/2001JA000299, 2002. 